\title{
An Efficient Technique for the Analysis of Reflectarrays
}

\author{
UDK 621.396.677.8
}

IFAC 4.3 .2

Original scientific paper

In this paper, we present an efficient technique based on the extension of the Adaptive Integral Method (AIM) that allows the full-wave analysis of microstrip reflectarrays. The reflectarray patches can have arbitrary shape and orientation and are modelled with subdomain triangular basis functions. The method makes use of a 2D-FTT/CG scheme, reducing the CPU time per iteration to $O(N \log N)$ and the memory requirement to $O(N)$.

Key words: numerical methods, reflectarrays

\section{INTRODUCTION}

Accurate and efficient electromagnetic simulations are essential for the analysis and the design of reflectarrays. Typically, however, a reflectarray is a large-scale electromagnetic problem and it is often necessary to employ a large number of unknowns for its solution. For the conventional MoM, whether in the spectral or spatial domain, the memory requirement is always proportional to $O\left(N^{2}\right)$, where $N$ denotes the number of unknowns. This requirement can easily become prohibitive even on the most powerful computers. Even if the memory permits, the computing time can, however, become very excessive. Recently, a few techniques, based on a modification of the classical method of moments (MoM), have been proposed for the analysis of electrically large microstrip structures [1-3]. These techniques allow a fast evaluation of the reaction integral and, when an iterative solver is used, a fast matrix-vector multiplication.

Here, a new technique is presented, called MultiLayer Adaptive Integral Method (MLayAIM) that is highly suitable for the analysis of multilayered structures, such as reflectarrays, which present large planar metallizations and a few vertical conductors (Figure 1). In particular, the MoM solution technique is applied to the surface integral equation, and the matrix equation is $\mathbf{Z} I=V$. The impedance matrix $\mathbf{Z}=\mathbf{Z}^{e}+\mathbf{Z}^{p}$ is then divided into the sum of a non-planar (external) matrix $\mathbf{Z}^{e}$ and a planar-matrix $\mathbf{Z}^{p}$. The planar matrix represents

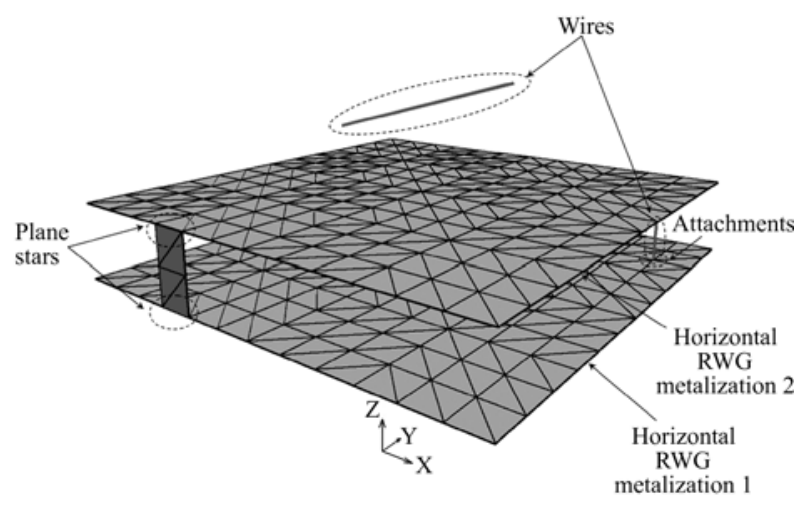

Fig. 1 Geometry of the problem

the interaction relevant to those basis functions that lie on a $z$-constant plane. Especially in a reflectarray analysis, these basis functions represent the majority; hence, the external matrix $\mathbf{Z}^{e}$ is a very sparse matrix and its storage does not present any problem. As the classical Adaptive Integral Method (AIM) [4], the efficiency is then achieved by splitting the reaction integral domain of the planar part into a near-interaction (strong) region and a far-interaction (weak) region, based on an appropriate choice of the error of the reaction integral terms. Hence, the planar impedance matrix $\mathbf{Z}^{p}$ is further divided into the sum of a strong- and a weak-matrix, i.e., $\mathbf{Z}^{p}=\mathbf{Z}^{s}+\mathbf{Z}^{w}$, where $\mathbf{Z}^{s}$ represents the (strong) near-field interaction and $\mathbf{Z}^{w}$ the (weak) non-near-field interaction. The near-interaction region requires that several reaction integrals have 
to be evaluated and stored in the strong-matrix $\mathbf{Z}^{s}$, which is a sparse matrix. Concerning the weak-matrix, use has been made of a set of auxiliary basis function supported by a two-dimensional Cartesian grid. This allows us to operate a canonical grid expansion of the exact weak-matrix elements into a series of translationally invariant terms. The so rewritten impedance matrix is then applied in a conjugate gradient solution of the matrix equation $\left(\mathbf{Z}^{e}+\mathbf{Z}^{s}+\mathbf{Z}^{w}\right) V=I$ with the required matrix-vector products performed rapidly due to the sparse nature of the external- and strong- matrices, and, for the weak-matrix, the use of the fast 2D Fourier transform (2D-FFT) and its inverse. When more than one planar metallization is present geometrical domain decomposition is used and a parallel implementation of the algorithm is feasible.

\section{FORMULATION}

Consider an arbitrarily microstrip structure consisting of $N_{m}$ metallized layers in a multilayer medium. Each layer is characterized by relative permittivity $\varepsilon_{r i}$, relative permeability $\mu_{r i}$, and thickness $h_{i}$. As well known, the equivalent currents $\overrightarrow{J_{s}}$ relevant to the microstrip structure can be found by solving the following MPIE [5]

$$
\hat{n} \times \vec{E}^{i}=j \omega \hat{n} \times\left\langle\underline{\underline{G}}^{A}, \vec{J}_{s}\right\rangle-\frac{1}{j \omega} \hat{n} \times \vec{\nabla}\left\langle G^{\Phi}, \vec{\nabla}^{\prime} \cdot \vec{J}_{s}\right\rangle
$$

where $\underline{G}^{A}$ and $G^{\Phi}$ are the Green's function for the vector and scalar potential, respectively. The notation $\langle$,$\rangle is used for integrals of dot products of two$ functions separated by the comma over their common spatial support. To solve the integral equation (1), one first subdivides the surface $\mathrm{S}$ of the metallic structure into small triangular patches, and the unknown current is expanded by using a suitableset of basis functions $\overrightarrow{J_{n}}(\vec{r})$ (e.g., the Rao-Wilton-Glisson (RWG) basis functions [6]), i.e.,

$$
\vec{J}_{n}(\vec{r})=\sum_{n=1}^{N} I_{n} \vec{j}_{n}(\vec{r})
$$

where $I_{n}$ are unknown coefficients and $N$ the number of interior edges (unknowns). Then, the MoM solution technique is applied to the surface integral equation (1), and the matrix equation is $\mathbf{Z} I=$ $=\left(\mathbf{Z}^{e}+\mathbf{Z}^{p}\right) I=V$. The external matrix $\mathbf{Z}^{e}$ is a sparse matrix and for its evaluation a classical MoM formulation is used. Hence, in the following, we will focus our attention on the planar-matrix $\mathbf{Z}^{p}$ evaluation only.
Let $P$ be the number of metallizations on which the planar part of the structure lies, each element $\boldsymbol{Z}_{m n}^{p}$ of the planar-matrix $Z^{p}$ can be expressed, assuming the Galerkin discretization scheme, as follows

$$
\begin{aligned}
& Z_{m n}^{p}=j \omega \iint_{S} \vec{j}_{m}(\vec{r}) \cdot \iint_{S^{\prime}} G_{p p^{\prime}}^{a}\left(\vec{r}, \vec{r}^{\prime}\right) \cdot \vec{j}_{n}\left(\vec{r}^{\prime}\right) \mathrm{d} \vec{r}^{\prime} \mathrm{d} \vec{r}+ \\
& +\frac{1}{j \omega} \iint_{S} \vec{\nabla} \cdot \vec{j}_{m}(\vec{r}) \iint_{S^{\prime}} G_{p p^{\prime}}^{\Phi}\left(\vec{r}, \vec{r}^{\prime}\right) \vec{\nabla}^{\prime} \cdot \vec{j}_{n}\left(\vec{r}^{\prime}\right) \mathrm{d} \vec{r}^{\prime} \mathrm{d} \vec{r}
\end{aligned}
$$

where the basis function $\vec{J}_{n}(\vec{r})$ lies on the $p^{\prime}$ metalization, while $\overrightarrow{J_{m}}(\vec{r})$ on the $p$ metallization. $G^{a} p p^{\prime}$ and $G_{p p^{\prime}}^{\Phi}$ are the dyadic Green's function and the scalar potential, respectively, due to the interaction between the metallization $p$ and $p^{\prime}$. It is worth noting that to solve the planar problem by using the formulation proposed in [7] it is sufficient to evaluate the $x x$-component $G_{p p^{\prime}}^{a}$ of the dyadic Green's function.

As already mentioned, the planar-matrix $\mathbf{Z}^{p}$ can be written as the sum of the near-field (strong) $\mathbf{Z}^{s}$ and the far-field (weak) $\mathbf{Z}^{w}$ matrices. The former one is calculated $\mathbf{Z}^{s}=\mathbf{Z}^{p}-\mathbf{Z}^{w}$ similarly as in [4], and is the only one that we have to store. Concerning the weak-matrix, we introduce a set of auxiliary basis functions $\vec{\psi}_{n}(\vec{r})$ and $\vec{\psi}_{n}^{d}(\vec{r})$ which produce a good approximation of the field radiated by a RWG basis function at a large distance. In particular, for each RWG basis function lying on a plane $z=z_{p}$, we chose a set of $L=(M+1)^{2}$ point-like current elements located at the nodes of a regular Cartesian two-dimensional grid, parallel to the $x, y$ plane and located at $z=z_{p}$, i.e.,

$$
\begin{gathered}
\vec{j}_{n}(\vec{r}) \simeq \vec{\psi}_{n}(\vec{r})=\sum_{i=1}^{L} \vec{\Lambda}_{n, i} \delta\left(\vec{r}-\vec{r}_{i}\right) \\
\vec{\nabla} \cdot \vec{j}_{n}(\vec{r}) \simeq \psi_{n}^{d}(\vec{r})=\sum_{i=1}^{L} \Lambda_{n, i}^{d} \delta\left(\vec{r}-\vec{r}_{i}\right)
\end{gathered}
$$

where $\vec{\Lambda}_{n, i}, \Lambda_{n, i}^{d}$ are the translation coefficients of the expansion and $\vec{r}_{i}$ is the position vector of the $i$-th grid node. The translation coefcients are chosen so as to reproduce the first $(M+1)^{2}$ multipole moments of the original basis function [4], i.e., for $0 \leq q_{1}, q_{2} \leq M$,

$$
\begin{gathered}
\sum_{i=1}^{L} \vec{\Lambda}_{n, i}\left(x_{n, i}-x_{c}\right)^{q_{1}}\left(y_{n, i}-y_{c}\right)^{q_{2}}= \\
=\iint_{T_{n}} \vec{f}_{n}\left(x, y, z_{p}\right)\left(x-x_{c}\right)^{q_{1}}\left(y-y_{c}\right)^{q_{2}} \mathrm{~d} x \mathrm{~d} y
\end{gathered}
$$




$$
\begin{gathered}
\sum_{i=1}^{L} \Lambda_{n, i}^{d}\left(x_{n, i}-x_{c}\right)^{q_{1}}\left(y_{n, i}-y_{c}\right)^{q_{2}}= \\
=\iint_{T_{n}} \vec{\nabla} \cdot \vec{f}_{n}\left(x, y, z_{p}\right)\left(x-x_{c}\right)^{q_{1}}\left(y-y_{c}\right)^{q_{2}} \mathrm{~d} x \mathrm{~d} y
\end{gathered}
$$

where, to minimize the numerical roundoff errors, $x_{c}, y_{c}, z_{p}$ are the center of the RWG basis function support. The set of auxiliary basis functions allows us to approximate each element of the weak matrix as

$$
\begin{gathered}
Z_{m n}^{w}=j \omega \sum_{j=1}^{L} \sum_{i=1}^{L} \vec{\Lambda}_{m, j} \cdot \vec{\Lambda}_{n, i} G_{p p^{\prime}}^{a}\left(z_{p}, z_{p^{\prime}},\left|\vec{\rho}_{i}-\vec{\rho}_{j}\right|\right)+ \\
+\frac{1}{j \omega} \sum_{j=1}^{L} \sum_{i=1}^{L} \Lambda_{m, j}^{d} \Lambda_{n, i}^{d} G_{p p^{\prime}}^{\Phi}\left(z_{p}, z_{p^{\prime}},\left|\vec{\rho}_{i}-\vec{\rho}_{j}\right|\right) .
\end{gathered}
$$

By sorting the RWG basis functions with respect to the $P$ planar metallization it is possible rewrite the weak matrix $\mathbf{Z}^{w}=\left\{\mathbf{Z}_{p p^{\prime}}^{w}\right\}$ as a $P \times P$ blocks matrix with

$$
\begin{gathered}
\mathbf{Z}_{p p^{\prime}}^{w}=j \omega\left[\Lambda_{p}^{x}\right]^{T} \mathbf{G}_{p p^{\prime} \Lambda_{p^{\prime}}^{a}+}^{x} \\
+j \omega\left[\Lambda_{p}^{y}\right]^{T} \mathbf{G}_{p p^{\prime}}^{a} \Lambda_{p^{\prime}}^{y}+\frac{1}{j \omega}\left[\Lambda_{p}^{d}\right]^{T} \mathbf{G}_{p p^{\prime}}^{\Phi} \Lambda_{p^{\prime}}^{d}
\end{gathered}
$$

where $\Lambda_{p}^{x}, \Lambda_{p}^{y}$ and $\Lambda_{p}^{d}$ are sparse matrices with each row containing only $(M+1)^{2}$ nonzero elements. Considering the unknown vector relevant to the weak part composed by $P$ vectors as

$$
\underline{I}^{w}=\left[\underline{I}_{1}^{w} ; \underline{I}_{2}^{w} ; \ldots ; \underline{I}_{p}^{w}\right]
$$

and by employing a conjugate gradient (CG) method as iterative solver, one can write the matrixvector multiplication $V^{w}=\mathbf{Z}^{w} I^{w}$ as

$$
\begin{gathered}
V_{p}^{w}=\left[\Lambda_{p}^{x}\right]^{T} \sum_{p^{\prime}=1}^{N_{p}} \mathbf{G}_{p p^{\prime} \Lambda^{\prime}}^{a} \Lambda_{p^{\prime}}^{x} \underline{I}_{p^{\prime}}^{w}+ \\
+\left[\Lambda_{p}^{y}\right]^{T} \sum_{p^{\prime}=1}^{N_{p}} \mathbf{G}_{p p^{\prime}}^{a} \Lambda_{p^{\prime}}^{y} \underline{I}_{p^{\prime}}^{w}+\left[\Lambda_{p}^{d}\right]^{T} \sum_{p^{\prime}=1}^{N_{p}} \mathbf{G}_{p p^{\prime}}^{\Phi} \Lambda_{p^{\prime}}^{d} \underline{y}^{\prime}
\end{gathered}
$$

where $V^{w}=\left[V_{1}^{w} ; V_{2}^{w} ; \ldots ; V_{P}^{w}\right]$. It is worth noting that $\mathbf{G}_{p p^{\prime}}^{a}$ and $\mathbf{G}_{p p^{\prime}}^{\Phi}$ are Toeplitz block matrices and we can evaluate the matrix-vector products in (9) by using 2D-FFTs and their inverse.

Due to the sparsity of matrices $\mathbf{Z}^{e}, \mathbf{Z}^{s}$ and $\Lambda_{p}^{x}$, $\Lambda_{p}^{y}, \Lambda_{p}^{d}$ the memory requirement is proportional to $O(N)$. The CPU time per CG iteration is dominated by the 2D-FFT computation of the matrix-vector product, which, since the number of metalized planes is quite small (usually less than five), is proportional to $O\left(N \log _{2} N\right)$.

\section{RESULTS}

In this section we present some results obtained with the MLayAIM approach. Firstly, we applied the method to the analysis of the reflectarray presented in [8]. Figure 2 shows the reflectarray antenna with its primary source (i.e., a $12-18 \mathrm{GHz}$ feed horn sustained by three rigid plastic struts) and the reflectarray layout. In particular, the structure is a microstrip reflectarray consisting of 437 rectangular patches loaded with a centred slot, printed on a single layer substrate $(h=3.175 \mathrm{~mm}$, $\left.\varepsilon_{r}=2.17\right)$. The reflectarray is excited by an offset horn antenna and radiates a linearly polarized wave at broadside. Figure 3 shows the radiation patterns at $13 \mathrm{GHz}$ in the $\mathrm{H}-$ and E-planes. A good agreement between the measurements and the simulation can be observed except around $-30^{\circ}$. This disagreement can be attributed to the feed horn blockage and to the struts presence. Moreover, we can observe a shift of $0.7^{\circ}$ of the main lobe in the $\mathrm{H}$-plane, probably due to an antenna misalignment. As a matter of fact, this justifies the presence of the uncorrected measured cross-polar component in the E-plane (it should be zero due to the antenna symmetry). As a confirmation, once we simulated the radiation pattern in a plane squinted of $0.7^{\circ}$ with respect to the nominal E-plane we obtain a cross-polar component very close to that measured, as shown in Figure 3 (b).

Concerning the effort required to perform the numerical analysis, we used in average 200 unknowns to model each patch, with a total number of 87639 unknowns. By setting the conjugate gradient residual tolerance to $10^{-4}$ a computation time of 1 hour and 35 minutes on a PC Xeon $2.8 \mathrm{GHz}$ has been required for each frequency step. The computation time has been for the great majority
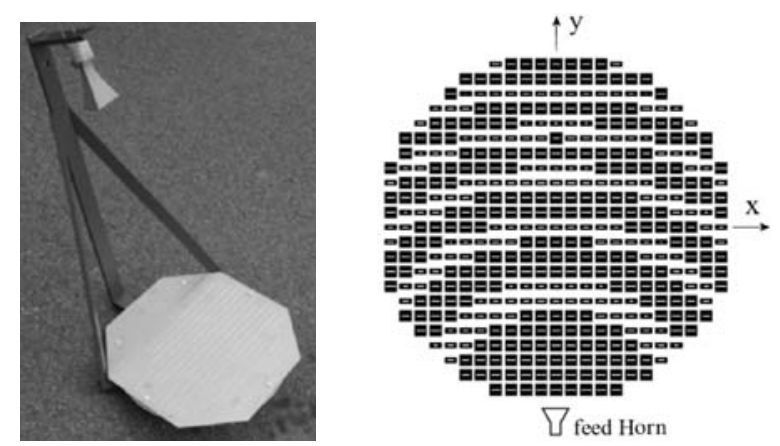

Fig. 2 Geometry of the reflectarray shown in [8] 

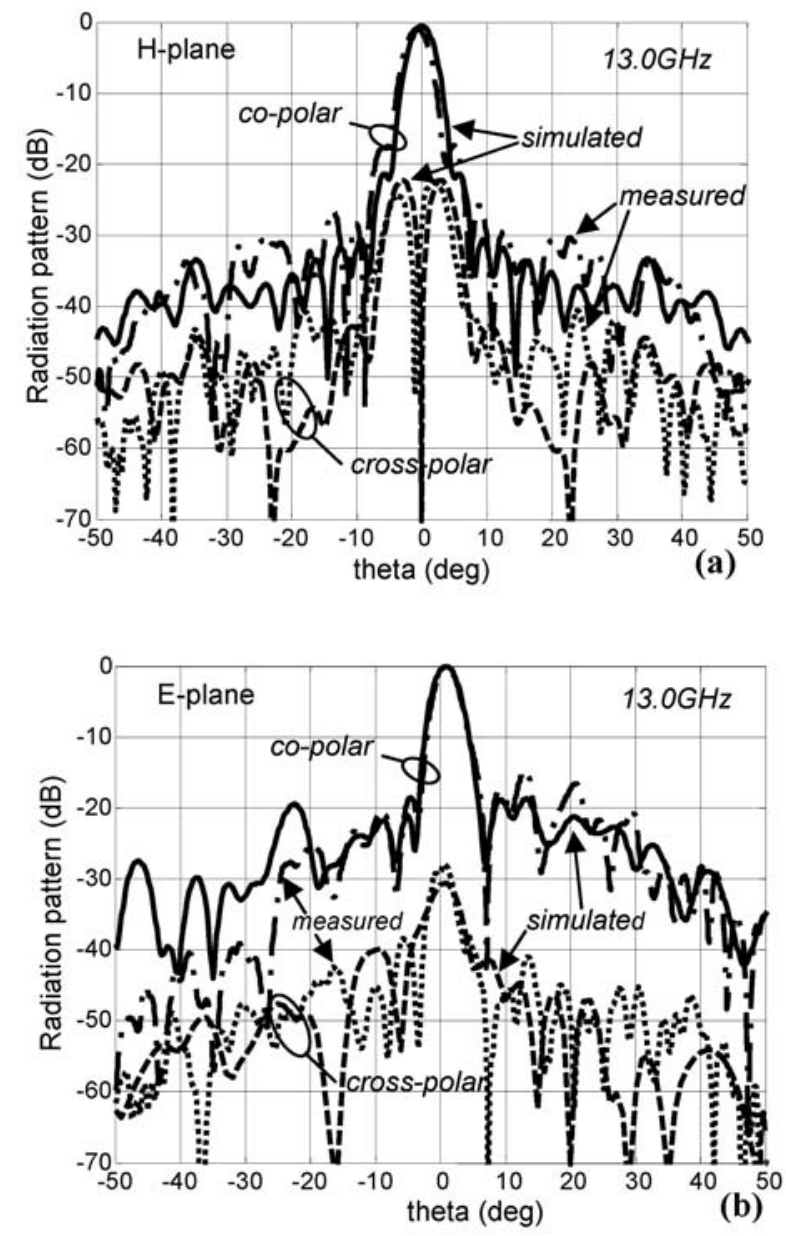

Fig. 3 Co-polar and cross-polar component of the radiation pattern at $13.0 \mathrm{GHz}$ : H-plane (a), E-plane (b)

due to the time required to perform the $\mathrm{CG}$ algorithm (i.e., 1 hour 16 minutes). When a MR preconditioner [9] is used the computation time is reduced to about 29 minutes.

A similar agreement between simulated and measured data has been observed also at $12 \mathrm{GHz}$ and $12.5 \mathrm{GHz}$, where measurements were available [10], even though with a faster numerical convergence.

Then, the proposed method has been applied to the analysis of the reflectarray presented in [11] that uses the radiating element shown in the inset of Figure 5.

A photo of the entire antenna is shown in Figure 4. It consists of $36 \times 36$ elements printed on a $h=$ $=1.6 \mathrm{~mm}$ grounded substrate $\left(\varepsilon_{r}=3, \tan (\delta)=\right.$ $=0.003)$. The reflectarray is illuminated by a standard horn that radiates the maximum field in the direction $\theta=15^{\circ}, \phi=0^{\circ}$.

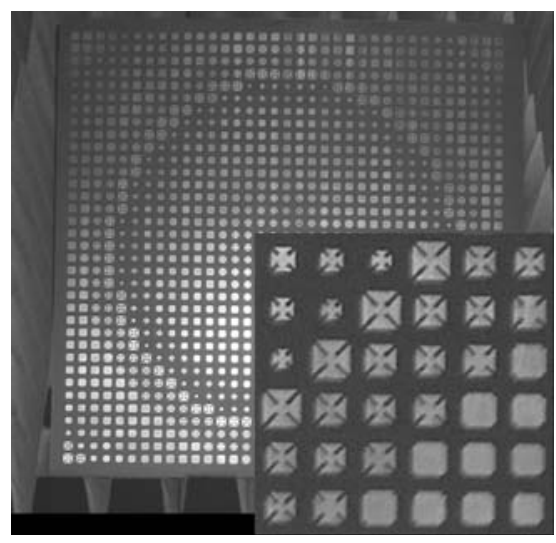

Fig. 4 Photograph of the manufactured reflectarray

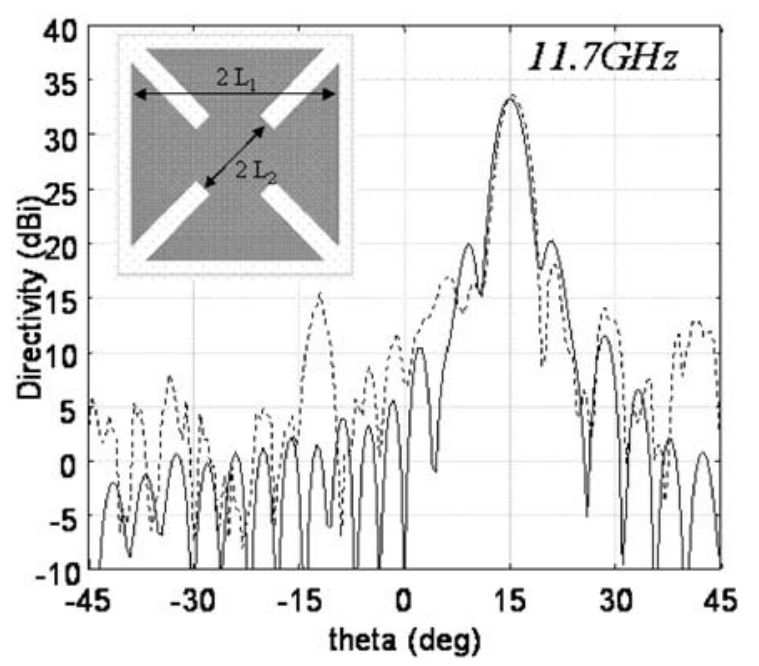

Fig. 5 Measured and simulated directivity pattern of the reflectarray of Figure 4. Dashed lines are relevant to measurements while continuous lines to the data obtained by using the MLayAIM

Figure 5 shows the antenna directivity in the E-plane. In particular, continuous lines represent the simulated data while dashed lines are relevant to the measured ones. As we can notice there is a quite good agreement between the measurements and the simulation. The disagreement observed especially around $\theta=-15^{\circ}$ is due to the influence of the open test range environment that has not be taken into account in the simulations.

Concerning the computational effort, we used 144181 unknowns to model the entire reflectarray. By setting the conjugate gradient residual tolerance to $10^{-4}$ a computation time of 2 hours and 52 minutes on a PC Xeon $2.8 \mathrm{GHz}$ has been required for each frequency step. For this specific case both MR and ILU(0) preconditioners failed. 


\section{ACKNOWLEDGEMENTS}

This work has been developed in the Sixth Framework Programme of the European Community within the Antenna Centre of Excellence (ACE).

\section{REFERENCES}

[1] J.-S. Zhao, W. C. Chew, C.-C. Lu, E. M, J. Song, Thin-stratified Medium Fast-multipole Algorithm for Solving Microstrip Structures. IEEE Trans. on Microwave Theory and Tech., MTT-46, 4, April, pp. 395 403, 1998

[2] F. Ling, C.-F. Wang, J.-M. Jin, An Efficient Algorithm for Analyzing Large-scale Microstrip Structures Using Adaptive Integral Method Combined with Discrete Complex-image Method. IEEE Microw. Theory and Tech., vol. MTT-48, pp. 832-839, 2000 .

[3] K. Zhao, J. F. Lee, A Single-level Dual Rank IE-QR Algorithm to Model Large Microstrip Antenna Arrays. IEEE Trans. Antennas and Propagat., vol. AP-52, pp. 2580-2585, 2004.

[4] E. Bleszynski, M. Bleszynski, T. Jaroszewicz, AIM: Adaptive Integral Method for Solving Large-scale Electromagnetic Scattering and Radiation Problems. Radio Science, vol. 5, pp. 1225-1251, 1996.
[5] J. R. Mosig, Arbitrarily Shaped Microstrip Structures and Their Analysis with a Mixed Potential Integral Equation. IEEE Trans. on Microwave Theory and Tech., MTT-36, 2, Feb., pp. 314-323, 1988.

[6] S. M. Rao, D. R. Wilton, A. W. Glisson, Electro-magnetic Scattering by Surface of Arbitrary shape. IEEE Trans. Antennas and Propagat., vol. AP-30, pp. 408-418, 1982.

[7] K. A. Michalski, J. R. Mosig, Multilayered Media Green's Functions in Integral Equation Formulations. IEEE Trans. Antennas and Propagat., vol. AP-45, pp. 508-519, 1997.

[8] D. Cadoret, A. Laisne, R. Gillard, L. Le Coq, H. Legay, Design and Measurement of New Reflectarray Antenna Using Microstrip Patches Loaded with Slot. IEE Electronics letters, vol. 41, pp. 623-624, 2005.

[9] P. De Vita, A. Freni, F. Vipiana, P. Pirinoli, G. Vecchi, Fast Analysis of Large Finite Arrays with a Combined MultiResolution - SM/AIM Approach. To appear on IEEE Trans. Ant. Prop., Vol. 54, Dec. 2006.

[10] ..., http://www.antennasvce.org/Community/SoftLAB, Network of Excellence "Antenna Centre of Excellence (ACE) «.

[11] P. De Vita, A. Freni, G. L. Dassano, P. Pirinoli, R. E. Zich, Broadband Element for High-gain Single Layer Printed Reflectarray Antenna. Electronics Letters, vol. 43, n. 23, pp. 1247-1249, Nov. 2007.

Efikasna metoda analize reflektorskih nizova. U radu je opisana efikasna metoda analize zasnovana na proširenju adaptivne integralne metode (AIM) koja omogućuje punovalnu analizu mikrotrakastih reflektorskih nizova. Pločice, elementi reflektorskih nizova, mogu imati proizvoljni oblik i orijentaciju pa su modelirane trokutasnim baznim funkcijama s domenom na dijelu pločice. Metoda rabi 2D-FTT/CG shemu, i pri tome smanjuje potrebno vrijeme rada računala na $O(N \log N)$ i memorijske zahtjeve na $O(N)$.

Ključne riječi: numeričke metode, reflektorski nizovi

\section{AUTHORS’'ADDRESSES}

Francesca De Vita

e-mail: francesca.devita@unifi.it

Paolo De Vita

e-mail: paolo.devita@unifi.it

Alberto Di Maria

e-mail: dimaria@dii.unisi.it

Angelo Freni

e-mail: freni@unifi.it

Department of Electronics and Telecommunications

University of Florence

Via di S. Marta 3, 50139 Florence, Italy

Received: 2008-01-25 